\title{
Active regulation of altitude as a function of optical texture
}

\author{
JOHN M. FLACH, BRENT A. HAGEN, and JOHN F. LARISH \\ Institute of Aviation, University of Illinois, Urbana, Illinois
}

\begin{abstract}
Two empirical studies are reported that examine active regulation of altitude as a function of the type of ground texture. Three ground textures were examined: lines perpendicular to the direction of motion, lines parallel to the direction of motion, and the combination (i.e., square or checkerboard texture). Although subjects only controlled altitude, disturbances were introduced on three axes: vertical, lateral, and fore-aft. The results show a clear advantage for texture parallel to the direction of motion. However, in considering these results in the context of previous research on altitude control, the argument is made that there is no compelling evidence that suggests either parallel (splay) or perpendicular (density) texture is privileged with regard to altitude control. Rather, the most effective display for altitude control will be the one that best isolates the optical activity associated with changing altitude from the optical activity arising from other sources of disturbance (such as forward locomotion). Such a display will make it easier for the observer to distinguish and respond specifically to the disturbances of altitude.
\end{abstract}

Gibson, Olum, and Rosenblatt (1955) argued that " motion perspective" provided "a basis for the judgments required for the control of locomotion"' (p. 385). Motion perspective refers to the flow of texture elements within the optic array of a moving observer. The research reported here focused on one aspect of the visual control of locomotion-regulation of altitude. Two sources of information within the optic array were examined: optical splay angle and global optical density (or optical depression angle). We begin by presenting an analysis of the optical geometry to show the linkage between altitude and optical splay angle and optical depression angle. Next, we review previous research where there is currently debate about the relative efficacy of these two sources of information. We then present two empirical studies that examined these two sources of information in an altituderegulation task. Finally, we attempt to integrate our results with those from previous studies and discuss the theoretical and practical implications.

This research was funded by a grant to the Aviation Research Laboratory at the University of nlinois from the NASA Rotocraft Human Factors Research Branch (Contract No. NASA NAG 2-308). Sandra Hart was contract monitor. The experiments were conducted at the Engineering Psychology Research Laboratory. Equipment used was obtained from an instrumentation grant from the Department of Defense administered by the Army Research Office. The principal author was supported by a grant from the Air Force Office of Scientific Research (AFOSR-91-0151) during the final preparation of this manuscript. Special thanks to Walt Johnson (NASA) and Lawrence Wolpert (LTSI) for helpful discussions during the course of this research. Thanks also to Jonathan Sivier for developing the software for this research. Correspondence should be addressed to J. M. Flach, Psychology Department, 309 Delman Hall, Wright State University, Dayton, OH 45435 (e-mail: jflach@falcon.aamrl.wpafb.af.mil).

\section{OPTICAL ANALYSIS}

The term optical splay was introduced by Warren (1982). Warren cites Biggs (1966), who noted that when an observer maintains a constant distance to a line on the ground plane (e.g., the curb of the road), despite the shifting optical positions of the individual points composing the line, the optical position of the line was invariant. For a straight line parallel to the direction of motion, the invariant optical position can be defined in terms of the angle at the vanishing point formed by the line and a second line perpendicular to the horizon along the ground trace of forward motion, as shown in Figure 1. Thus, it can be defined by the equation:

$$
S=\arctan \left(Y_{b} / z\right) \text {, }
$$

where $S$ is the splay angle, $Y_{g}$ is the lateral displacement of the line from the perpendicular, and $z$ is the altitude (eyeheight) of the observer. This equation describes the projection onto the frontal plane for an observer moving parallel to the ground. For rectilinear motion over a flat ground plane, splay angle will be constant when altitude is constant.

The rate of change in splay angle is specified by the following equation:

$$
\dot{S}=(-i / z) \cos S \sin S+\left(\dot{Y}_{g} / z\right) \cos ^{2} S
$$

The first term $[(-\dot{z} / z) \cos S \sin S]$ indexes change in splay as a function of changes in altitude. The negative sign indicates that as altitude $(z)$ decreases, splay angle will increase, and vice versa. The term $(-i / z)$ specifies fractional change in altitude or change in altitude scaled in eyeheights. This term indicates that the relation between change in altitude and change in splay angle will depend on the initial altitude. At high altitudes (large $z$ ), any given 


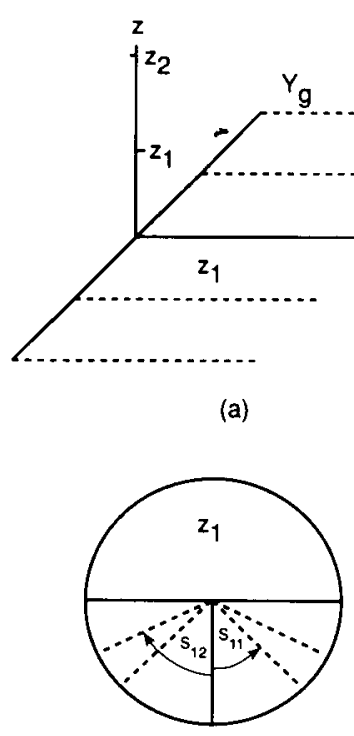

(b)
$S_{11}=\tan ^{-1}\left(Y_{g 1} / z_{1}\right)=1 / 1=45^{\circ}$

$S_{12}=\tan ^{-1}\left(Y_{g 2^{2}} / z_{1}\right)=2 / 1=63.4^{\circ}$ $S_{21}=\tan ^{-1}\left(Y_{g 1} / Z_{2}\right)=1 / 2=23.6^{\circ}$ $S_{22}=\tan ^{-1}\left(Y_{g 2} / z_{2}\right)=2 / 2=45^{\circ}$

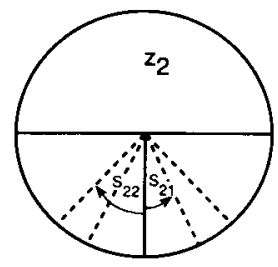

(c)
Figure 1. An illustration of optical splay angle for parallel texture elements at two distances from the line of motion $\left(Y_{81}\right.$ and $\left.Y_{82}\right)$ and for two eyeheights (altitudes) $\left(z_{1}\right.$ and $\left.z_{2}\right)$; (b) shows the perspective view from eyeheight $=z_{1}$; (c) shows the perspective view from eyeheight $=z_{2}$.

change in altitude would result in a smaller change in splay angle than when initial altitude was lower. As noted by Warren (1988), "sensitivity of the display [optical splay rate] varies inversely with altitude: the lower the altitude, the more change in visual effect for equivalent altitude change commands. At very low altitudes this optical activity is dramatic even optically violent"' (p. A121). The $(-\dot{z} / z)$ term is independent of optical position. It scales the rate of change for every splay line in the field of view. For this reason, it has been termed "global perspectival splay rate" (Wolpert, 1987). The sine and cosine terms index the dependence of splay rate on optical position. For splay lines with $0^{\circ}$ splay angle (perpendicular to horizon at the expansion point) and $\pm 90^{\circ}$ splay angle (the horizon), the rate of change will be zero. From these minima, the rate of change in splay angle for a given fractional change in altitude will increase to a maximum at a splay angle of $\pm 45^{\circ}$.

The second term in Equation $\left.2\left[\dot{Y}_{g} / z\right) \cos ^{2} S\right]$ indexes change in splay as a function of change in lateral distance $\left(\dot{Y}_{g}\right)$ from the observer to the line element such as might result from a lateral movement of the observer. For straight ahead, forward motion there is no change in lateral distance $\left(\dot{Y}_{B}=0\right)$ and this term has no impact on the optical splay angle. For this reason, this term has not typically been included in analyses of splay. However, lateral displacements have sometimes been included in the events that have been simulated to study altitude control. Thus, it is important to understand the effects from this term. The first half of the term $\left[\left(\dot{Y}_{g} / z\right)\right]$ specifies lateral displacements scaled in eyeheights. Changes in lateral distance will result in proportional changes in splay angle. The second half of the term $\left(\cos ^{2} S\right)$ indicates the effect of optical position on change in splay angles. This term decreases from a maximum for the texture line directly below the observer $\left(S=0^{\circ}\right)$ to a minimum at the horizon $\left(S= \pm 90^{\circ}\right)$.

Global optical density has been defined by Warren (1982) as "the number of ground elements required to span one eyeheight distance." Formally, optical density (OD) is defined as follows:

$$
\mathrm{OD}=z / g,
$$

where $z$ is the altitude of the observer and $g$ is the extent of a ground texture element (e.g., the distance between grid lines in Figure 2b). Thus, for a constant texture size ( $g=$ constant), changes in altitude $(z)$ will result in proportional changes in optical density. As altitude increases, optical density will increase, and vice versa. Optical density is a global optical variable in that it is an index of the general optical "sparseness" within the field of view. It is not associated with a specific texture element as is optical splay. However, in attempts to create a visual stimulus that isolates global optical density from optical splay, experimenters have used visual fields textured only by lines perpendicular to the direction of travel (e.g., Johnson, Bennett, O'Donnell, \& Phatak, 1988; Johnson, Tsang, Bennett, \& Phatak, 1989; Wolpert, 1983, 1988; Wolpert \& Owen, 1985; Wolpert, Owen, \& Warren, 1983). This type of texture can be seen in Figure $2 b$.

To understand the control of altitude with regard to this kind of texture, it is important to examine the optical activity of these perpendicular lines as a function of changes in altitude. Position of the perpendicular line within the visual field can be specified in terms of the angular distance below the horizon $(\delta)$ as shown in Figure 3. This angle, which will be termed optical depression angle, can be expressed as follows:

$$
\delta=\arctan \left(z / x_{\mathbf{g}}\right),
$$

where $z$ is altitude of the observer and $x_{g}$ is the distance

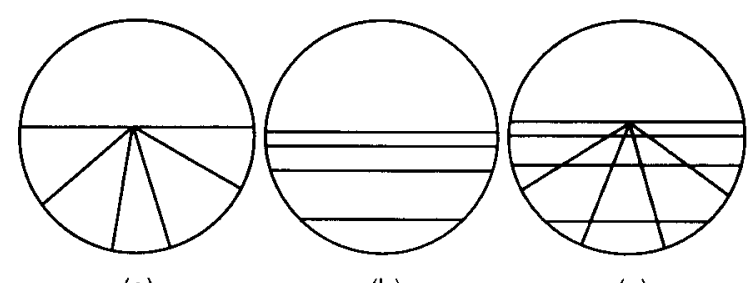

(a)

(b)

(c)

Figure 2. Three kinds of ground texture: (a) parallel to the direction of motion; (b) perpendicular to the direction of motion; and (c) square texture. 


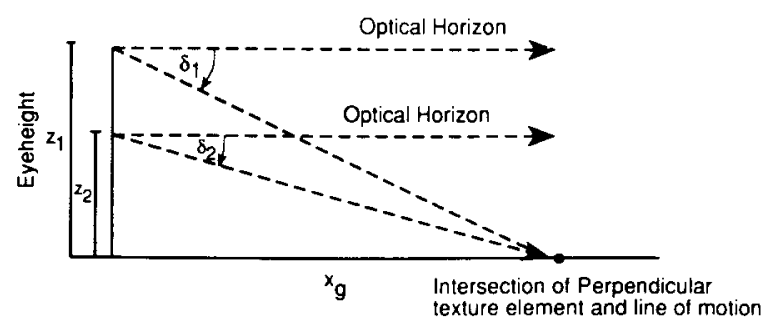

Figure 3. Illustrates the relation between depression angle $(\delta)$ of perpendicular texture element and eyeheight.

on the ground from the observer to the specific perpendicular line.

For rectilinear motion over a flat ground plane, the rate of change of the optical depression angle will be:

$$
\dot{\delta}=(\dot{z} / z) \cos \delta \sin \delta-\left(\dot{x}_{z} / z\right) \sin ^{2} \delta .
$$

The first term $[(z / z) \cos \delta \sin \delta]$ shows the contribution of changes in the observer's altitude on the optical depression angle. The relation between depression angle and altitude is qualitatively identical to the relationship between splay angle and altitude, with the exception of sign. As with splay angle, the rate of change in depression angle scales with fractional changes in altitude. Also, as with splay angle, the rate of change in depression angle as a function of change in altitude will be dependent on optical position. Rate of change in depression angle will be zero at depression angles of $90^{\circ}$ (directly below the observer) and $0^{\circ}$ (horizon) and will be maximum at a depression angle of $45^{\circ}$.

The difference in sign indicates that splay angle increases with decreasing altitude, whereas depression angle decreases with decreasing altitude. This difference can be misleading. Splay angle is indexed to the line of sight, but depression angle is indexed to the horizon. Actually, both splay angle and depression angle are components of an expansion of texture that is associated with approach to a surface. The term $(z / z)$ is a measure of the rate of expansion. As Gibson et al. (1955) note in their description of optical flow:

Ground speed and altitude are not ... independently determined by optical information. A more rapid flow pattern may indicate either an increase in speed or a decrease in altitude. Length of time before touching down, however, seems to be given by the optical information in a univocal manner. (p. 382)

This last statement anticipates a more complete analysis by Lee $(1976,1980)$. This analysis shows that the time to contact (tau) with a surface is specified as the inverse of the rate of optical expansion, which in our case will be $(z / z)$. Thus, splay and depression angle can be seen as isolated components from a global optical expansion resulting from approach toward a ground surface.

The second term in Equation $5\left[\left(\dot{x}_{g} / z\right) \sin ^{2} \delta\right]$ indexes changes in depression angle as a result of forward motion of the observer. In the first part of this term, $\dot{x}_{z}$ is proportional to the speed of the observer. The term $\left(-\dot{x}_{g} / z\right)$ is forward speed scaled in eyeheights. Warren (1982) called this factor global optical flow rate. Gibson et al.'s (1955) analysis of motion perspective shows that the speed of all points in the visual field is scaled with respect to global optical flow. Speed of texture flow is directly proportional to the forward speed of the observer and inversely proportional to the altitude of the observer. Larish and Flach (1990) reported an empirical investigation of the role of global optical flow rate in judgments of speed of self-motion. The remaining part of this term $\left(\sin ^{2} \delta\right)$ accounts for changes in depression rate due to optical position. The consequence of this term is that the larger the initial depression angle (the lower the line is in the field of view), the greater will be the rate of change of depression angle for a given speed of observer movement. Rate of change of depression angle for an observer moving forward at a constant speed and constant altitude will be minimum at the horizon and will increase to a maximum at a point directly below the observer.

\section{PREVIOUS RESEARCH}

Wolpert et al. (1983) compared observers' ability to detect loss in altitude in a simulation of flight with constant forward speed using three types of texture, as shown in Figure 2: parallel (meridian) texture, perpendicular (lateral) texture, and square (checkerboard) texture. Parallel texture was chosen to isolate the information available from optical splay, and perpendicular texture was chosen to isolate the information available from global optical density. The results indicated that observers were better able to detect loss in altitude with parallel texture (optical splay only) than with either square texture (splay and depression angle) or perpendicular texture (optical depression angle only). A number of these studies are summarized by Wolpert (1983, 1987; Wolpert \& Owen, 1985; Wolpert et al., 1983). Wolpert (1987) notes that in these studies "loss in altitude scaled in eyeheights proved to be the functional variable, performance improving over increasing levels of that variable. In contrast, ground-unitscaled loss in altitude showed a minimal effect over the different levels"' (p. 24). Because the rate of change of optical splay is directly related to change of altitude scaled in eyeheights (Equation 2), although rate of change of optical density is related to change of altitude scaled in ground units (Equation 3), splay was thought to be the more effective source of information for regulating altitude. This conclusion must be reconsidered in light of the optical analyses above, which show that both optical splay angle (Equation 2) and optical depression angle (Equation 5) are proportional to change in altitude scaled in eyeheights.

This strategy of isolating perpendicular and parallel texture in visual displays has also been used in research on slant perception (Attneave \& Olson, 1966; Gillam, 1968, 1970). Texture parallel to the line of motion (Figure 2a) isolates the perspective gradient (i.e., convergence). Tex- 
ture perpendicular to the line of motion (Figure 2b) isolates the compression gradient. Square texture (Figure 2c) contains both perspective and compression gradients. Gillam (1968) found that judgment of slant was similar for texture parallel to the line of motion (convergence) and square texture, but judgments were significantly lower for perpendicular texture (compression). Attneave and Olson found that when convergence and compression conflicted, the judged direction of slant was determined by the direction of convergence. Cutting and Millard (1984) summarize research on slant perception, noting that "it is generally known that the perspective gradient is more potent than compression (e.g., Rosinski \& Levine, 1976; Vickers, 1971) for the perception of a flat surface receding in the distance"' (p. 201). Thus, the parallel texture (splay angle) appears to dominate for both judgments of altitude (Wolpert et al., 1983) and judgments of slant. Note that slant was not varied in any of the experiments discussed in this paper. All events simulated flight over a flat ground plane (slant $=90^{\circ}$ ).

Johnson et al. (1989) employed a strategy similar to that of Wolpert et al. (1983) to isolate the optical information available for control of altitude. They used three texture types: parallel (meridian) texture only, which isolates optical splay angle; perpendicular (latitude) texture (with a single meridian line roadway to indicate flight path), which was intended to isolate optical depression angle; and square texture, which contains both optical splay and optical depression information. Wolpert et al. measured performance in a passive psychophysical judgment task, and Johnson et al. measured performance in an active control task. Johnson et al. introduced disturbances in both the vertical and lateral axes. Subjects were to minimize the effects of the vertical disturbance using a single-axis velocity control. Subjects' control actions had no effect on the lateral disturbance. The lateral (side-to-side) disturbance was introduced to prevent subjects from using a strategy in which local information such as the position of a meridian texture line on the bottom of the display (distance from corner of rectangular display) was used to control altitude. In apparent contradiction to the results of Wolpert et al., Johnson et al. found superior performance (lower tracking error) with the perpendicular (optical depression angle) and square textures. Highest tracking error was found for the meridian display, which contained the most salient information with regard to splay.

Two aspects of the Johnson et al. (1989) study should be noted. First, the lateral disturbance was transparent with respect to the perpendicular texture (optical depression angle only). The lateral disturbance affected only the parallel texture and thus could only be seen in the parallel and checkerboard textures. ${ }^{1}$ Second, the "roadway" in the lateral display may have provided some splay information. If the roadway had always been directly under the observer (splay angle $=0^{\circ}$ ), then no change in splay angle would have resulted from changing altitude. However, because of the lateral disturbance, the roadway would have provided splay information at those times when the observer was off course because of the lateral disturbance. We grant, however, that it may have been difficult for observers to disambiguate the changes in splay associated with changes in altitude from those associated with changes in lateral position (Equation 2).

A second study by Johnson et al. (1988) ${ }^{2}$ examined active control of altitude in a hover task. In this task, Johnson et al. included disturbances on three axes: altitude, lateral (visible only in parallel texture), and fore-aft (visible only in perpendicular texture). Performance was examined for numerous texture types, four of which were of particular interest for the present discussion: parallel, perpendicular, square, and dot. The results showed equivalent performance (both in terms of tracking error and correlated control power) for the perpendicular, square, and dot textures. Performance with the parallel (splay only) texture showed greater tracking error and lower correlated control power. Again, this result is in apparent contradiction to the findings of Wolpert et al. (1983).

An important difference in the Johnson et al. (1988, 1989) studies was the inclusion of disturbances on axes other than the vertical axis. In the Wolpert et al. (1983) studies, disturbances only occurred on the vertical axis. Johnson et al. (1989) suggested that the poor control using parallel texture in their studies may be due "to people inappropriately attending to, and attempting to hold invariant local optical structures. Two such structures, window edge/meridian line intersection location and local splay angle, have been suggested as possible candidates" (p. 156). Holding such local structures invariant will be an effective strategy for altitude control if the only disturbances are in the vertical direction. The implication is that the superior performance for splay found by Wolpert et al. may have been due to local, two-dimensional, pictorial cues. Thus, there is some concern about whether these results will generalize to the control of altitude in a "real-world" (three-dimensional) flight-control task. To test this, Wolpert (1988) employed an active altituderegulation task with disturbances in altitude and roll (subjects only controlled altitude). Note that a roll disturbance affects the optical activity of both parallel and perpendicular texture. ${ }^{3}$ Results were consistent with the previous findings of Wolpert et al. "Altitude was better maintained over parallel texture than over square or perpendicular texture" (Wolpert, 1988, p. 17). Wolpert found that whether or not the roll disturbance was included had no effect.

Wolpert (1988) also included optical flow rate as a variable in his study. He found a performance decrement for increasing levels of global optical flow rate. This is consistent with previous research by Wolpert and Owen (1985). That study found that detection of descent over square texture deteriorated with increasing global optical flow rates $(0.25,0.50$, and 1.0 eyeheight $/ \mathrm{sec})$. This is interesting in light of the optical analyses presented earlier. Optical splay angle is independent of global opti- 
cal flow rate (Equations 1 and 2). However, depression angle is dependent on global optical flow rate (Equation 5). Global optical flow rate $\left(\dot{x}_{z} / z\right)$ changes as a function of altitude $(z)$. However, changes in global optical flow are not specific to altitude. Global optical flow rate is directly proportional to forward velocity $\left(\dot{x}_{s}\right)$ and inversely proportional to altitude $(z)$. This ambiguity had been previously noted in the optical analysis of Gibson et al. (1955).

It is interesting to note that the global optical flow rates examined by Wolpert (1988) were all greater than 1 eyeheight/sec. However, the optical flow rates examined by Johnson et al. $(1988,1989)$ ranged from 0 for the hover task to .25 eyeheight $/ \mathrm{sec}$. Thus, in the Johnson et al. studies the optical flow rates were far lower than in previous studies. One eyeheight/sec is approximately the speed of a person walking at a comfortable pace. Could an interaction between global optical flow and the other sources of information for altitude (optical splay angle and optical depression angle) account for the disparate findings described above?

One final observation: In each of the studies discussed above, the texture that isolated the most effective optical information (whether splay or depression angle) always yielded performance that was superior to (though not typically significantly superior to) the display that combined the two sources of information (checkerboard or dot texture). Wolpert et al. (1983) and Wolpert (1988) found that performance was better with parallel (splay only) texture than with checkerboard texture. Johnson et al. (1988, 1989) found that performance was better with perpendicular (depression angle only) texture than with checkerboard or dot textures. Also, Warren (1988) found that altitude control was superior with parallel texture only than with parallel plus superimposed dot texture. Why does the combination of multiple sources of information result in performance degradation?

Before we attempt to answer these questions, we will report two empirical studies on the regulation of altitude. These experiments employed disturbances on three axes similar to those used by Johnson et al. (1988), but instead of the hover task examined by Johnson et al., altitude was examined in the context of forward locomotion as in the studies of Wolpert et al. (1983) and Wolpert (1988). Global optical flow rates of 1 and 0.5 eyeheight/sec were used. These are intermediate between the levels used in previous studies.

\section{EXPERIMENT 1}

This experiment employed a methodology similar to that used by Johnson et al. (1988). Three different textures were used: parallel (splay only), perpendicular (depression angle only), and square texture (splay and depression angle). Subjects had active control of altitude only. However, disturbances were introduced on three axes: vertical (altitude), lateral (side-to-side), and variable head wind (fore-aft). Vertical displacements affected both parallel and perpendicular texture elements. Lateral displace- ments affected only parallel texture elements. The fore-aft disturbance was different than that used by Johnson et al. (1988). Johnson et al. simulated a hover task in which the fore-aft disturbance resulted in forward and backward displacements of the observer, whereas the present study simulated continuous forward motion with variable head winds. In both studies, this disturbance was invisible with respect to parallel texture. It was visible only when the perpendicular texture elements were present.

The disturbance was composed of three sets of functions, which were each composed of three sine waves at different, nonharmonically related, frequencies. Each set corresponded to one of the three axes (vertical, lateral, head wind). The frequencies chosen for the sine waves resulted in an interleaving of the disturbances in frequency space, as illustrated in Figure 4. This interleaving of disturbance frequencies allowed a microanalysis of the subjects' control actions into the power correlated with each of the three disturbances. Since the subjects' task was to control altitude, a relatively greater amount of control power was expected to appear at frequencies associated with the vertical disturbance. To the extent that optical activity associated with the lateral disturbance or variable head wind was mistaken for changes in altitude, relatively more power was expected at those frequencies. Thus, with parallel texture, more control power was expected at frequencies associated with the lateral disturbance, while
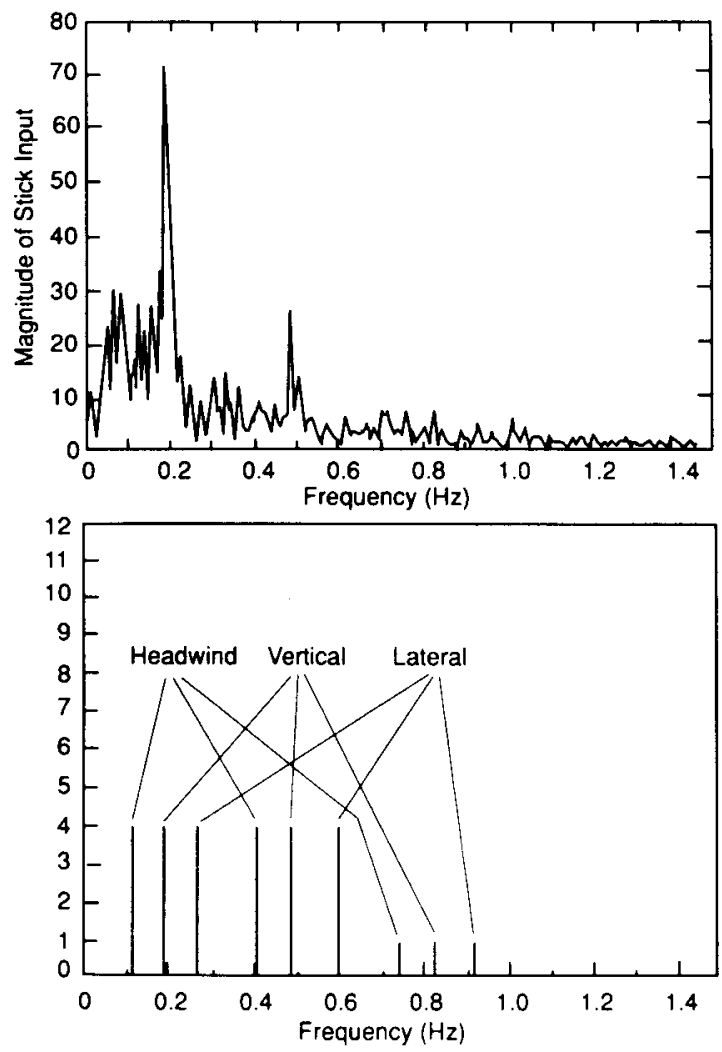

Figure 4. A typical frequency plot of control activity with disturbance frequencies highlighted below. 
with perpendicular texture, more power was expected at frequencies associated with the head-wind disturbance.

\section{Method}

Subjects. Nine University of Illinois undergraduate students participated in this experiment for course credit. All were male, were right-handed, and had normal or corrected-to-normal vision.

Design. The design can be viewed from a macroscopic or microscopic perspective. At the macroscopic level, performance was evaluated with respect to a trial. The dependent variables were mean altitude error, standard deviation of altitude, residual power (total stick power at noninput frequencies), and RMS stick input. These performance measures were evaluated with respect to the independent variables texture (parallel, perpendicular, or square) and dominant altitude frequency $(0.1099,0.1904$, or $0.2637 \mathrm{~Hz})$. The dominant altitude frequency was the frequency of the lowest frequency sine wave composing the vertical disturbance. Thus, with respect to the macroscopic variables, the design was a $3 \times 3$ factorial in which all variables were manipulated within subjects. Each subject received all nine combinations of texture and dominant altitude frequency. Each subject received a different viewing order, which was determined using a Latin square.

At the microscopic level, control power was evaluated at the frequencies associated with each disturbance set. The independent variables related to this performance measure were texture (parallel, perpendicular, or square), direction of disturbance (vertical, lateral, or head wind), and frequency of disturbance (low, medium, or high). These variables were manipulated within subjects in a 3 $\times 3 \times 3$ factorial design.

Apparatus. The displays were generated using a Silicon Graphics IRIS 3130 graphics system with a high-resolution $(1,024 \times 768$ pixel) color monitor (Silicon Graphics Model CM2073A). All displays were presented at an update rate of approximately 15 frames/sec. The subjects were seated in a darkened booth, approximately $60 \mathrm{~cm}$ from the screen. A piece of black poster board was placed over the front of the monitor. A $24.5-\mathrm{cm}$-diam, circular view port was cut in the board, and the centers of both the monitor and the view port were aligned. This resulted in a circular field of view of approximately $23^{\circ}$ of visual angle. The circular presentation format was used to reduce framing cues that might minimize the illusion of depth in the display (without depth, it would be debatable whether we were studying self-motion perception). This format also eliminated the rectangular corners that may have provided landmarks for local strategies for controlling altitude.

The event simulated was continuous forward motion with initial and mean global optical flow rate of 1 eyeheight/sec. ${ }^{4}$ Three textures were employed. Parallel texture was constructed of color bands parallel to the direction of motion. The distance across a texture element was 2 eyeheights. Thus, at the initial altitude, the splay angle associated with the edge of the middle texture element was $45^{\circ}$. Perpendicular texture was constructed of color bands perpendicular to the direction of motion. As with the parallel texture, the distance across a texture band was 2 eyeheights. These two textures were combined to create the checkerboard texture.
For the first $10 \mathrm{sec}$ of each trial, no disturbance was present so that the subjects could view the target altitude. At the end of this period, a beep sounded to indicate that the subject had control. Over the next $10 \mathrm{sec}$, the disturbances were ramped to their full value. Each trial continued for an additional 160 -sec period with full disturbance and control present. Data collection began at the beginning of this $160-\mathrm{sec}$ period and consisted of 2,048 points collected at a rate of $15 \mathrm{~Hz}$, for a duration of $136.53 \mathrm{sec}$.

Three different disturbances, identified by the dominant altitude frequency, were used. Each disturbance was composed of three sets of three frequencies each. Each set of frequencies affected a different axis (vertical, lateral, or head wind). Table 1 shows the frequencies that were used. The frequencies were chosen to be far enough apart to allow good resolution of the spectral analysis while avoiding harmonics. All disturbances were below $1 \mathrm{~Hz}$. The amplitude for the six lowest frequency sine waves was 0.19 eyeheight. The amplitude for the three highest frequency sine waves was 0.05 eyeheight. This is a gentle disturbance relative to many manualcontrol tasks. However, this level was chosen to avoid limitations due to motor systems and to avoid the need for lengthy training of subjects. We were interested in the response of naive subjects when asked to control altitude. Also, for this reason no feedback with respect to error was provided to the subjects.

The subjects controlled altitude by using a mouse. Control was limited to the vertical axis. Displacement of the mouse affected rate of change of altitude with a maximum descent (ascent) rate of 0.47 eyeheight/sec at the target altitude $(150 \mathrm{~m} / \mathrm{sec})$. Forward movements of the mouse caused a decrease in altitude, and backward movements caused an increase in altitude. A $1-\mathrm{cm}$ displacement resulted in a rate of descent (ascent) of 0.18 eyeheight/sec at the target altitude $(57.75 \mathrm{~m} / \mathrm{sec})$

Procedure. The subjects were instructed to maintain a constant altitude by using the mouse to regulate against vertical disturbances. The subjects participated for 2 days. The first day was used to familiarize the subjects with the task and apparatus. No data were collected for this day. Performance was measured on the second day. On each day, each of the nine combinations of texture and dominant altitude frequency were viewed twice in succession, for a total of 18 trials. The subjects were given brief rests between trials. No summary feedback about error was provided.

\section{Results}

Macroscopic. A $3 \times 3$ repeated measures analysis of variance (ANOVA) (texture $\times$ dominant altitude frequency) was performed for each of six macroscopic performance variables: mean altitude error, adjusted mean altitude error, standard deviation of altitude, adjusted standard deviation of altitude, residual power, and RMS control. For the adjusted measures of mean altitude and standard deviation, each trial's data was divided into 16 8.533-sec sets of 128 data points. The mean and standard deviation were calculated for each set of points, and the median was taken from the resulting 16 values. This mea-

Table 1

Dominant Altitude Frequencies and Frequencies of Disturbance Settings

\begin{tabular}{lccc}
\hline $\begin{array}{c}\text { Dominant } \\
\text { Altitude } \\
\text { Frequency }\end{array}$ & \multicolumn{3}{c}{ Frequency of Disturbance Settings } \\
\cline { 2 - 5 } & $\begin{array}{c}\text { Up-Down } \\
\text { (Vertical) }\end{array}$ & $\begin{array}{c}\text { Side-to-Side } \\
\text { (Lateral) }\end{array}$ & $\begin{array}{c}\text { Head-Wind } \\
\text { (Fore-Aft) }\end{array}$ \\
\hline Low $=0.1099 \mathrm{~Hz}$ & Low & Medium & High \\
Medium $=0.1904 \mathrm{~Hz}$ & Medium & High & Low \\
High $=0.2637 \mathrm{~Hz}$ & High & Low & Medium \\
\hline
\end{tabular}

Note-Sine-wave frequencies for frequency of disturbance settings: Low $=0.1099+0.4102$

$+0.7324 \mathrm{~Hz} ;$ Medium $=0.1904+0.4907+0.8102 \mathrm{~Hz} ; \mathrm{High}=0.2637+0.6006+0.9082 \mathrm{~Hz}$. 
sure reduced the effects of slow drifts away from the target altitude on the measure of standard deviation. This may be important because, unlike many tracking tasks where there is a continuously available fixed reference for the target, in this task the subject must remember what the target altitude was. Thus, during the trial the subjects may forget the original altitude reference and find themselves tracking a new altitude.

A significant main effect due to texture was found for mean altitude error $[F(2,16)=19.58, p<.001]$. The mean altitude error was significantly higher with the perpendicular (depression angle only) texture $(+0.66$ eyeheight) than with the parallel (splay angle only) texture $(+0.14$ eyeheight) or the square texture $(+0.22$ eyeheight). The difference between parallel and square textures was not significant. Note that for all textures mean altitude was greater than the target altitude (this is erring on the side of safety). No other main effects or interactions were found to be significant for this measure. An identical pattern of effects was found for adjusted mean altitude error.

Analysis of the standard deviation of altitude showed significant main effects for texture and for dominant altitude frequency. The main effect for texture $[F(2,16)=$ $53.32, p<.001]$ showed significantly larger deviations for perpendicular (depression angle only) texture (0.46 eyeheight) than for either parallel (splay angle only) texture $(0.20$ eyeheight) or square texture $(0.22$ eyeheight). The main effect for dominant frequency $[F(2,16)=7.34$, $p<.01]$ showed greater deviations when the dominant frequency was low (0.34 eyeheight) than when the dominant frequency was high ( 0.25 eyeheight). Again, essentially the same pattern of effects was found for the adjusted standard deviation measure.

For the residual power, which was an index of the amount of control power at noninput frequencies (this is the control activity that is not correlated with the disturbances), significant main effects were found for texture and dominant altitude frequency. The texture effect $[F(2,16)=9.38, p<.005]$ showed significantly more residual power with the perpendicular texture than with either the parallel or square textures, which were statistically equivalent for this measure. The effect of dominant altitude frequency $[F(2,16)=19.58, p<.001]$ showed significantly greater residual power for the lowest dominant altitude frequency than for either the medium or highest frequency, with no significant difference between the latter two.

The measure RMS control, which was a measure of overall control activity, resulted in significant main effects for both texture and dominant altitude frequency. The texture main effect $[F(2,16)=4.77, p<.025]$, when tested using Tukey's $H S D$, showed the perpendicular texture generating more activity than either the parallel or square textures, which were equivalent. The main effect for dominant altitude frequency $[F(2,16)=21.93$, $p<.001]$ displayed a significantly higher amount of activity for the lowest frequency, with no differences between the two higher frequencies.
Microscopic. Figure 4 shows a typical frequency plot of control activity. Note the spikes at the frequencies corresponding to the altitude disturbance. A $3 \times 3 \times 3$ repeated measures ANOVA (texture $\times$ disturbance $\times$ frequency) was performed for the correlated control power. The data used for this analysis were the control power at particular disturbance frequencies. This is the height of the spikes associated with disturbance inputs, as shown in Figure 4. This analysis resulted in a significant threeway interaction of texture $\times$ frequency $\times$ direction $[F(8,64)=2.45, p<.025]$. As can be seen in Figure 5, power was similar across textures and frequencies of disturbance for the lateral and head-wind disturbances. The difference appears to lie with the power associated with vertical (up-down) disturbances. The parallel and square textures both showed more power and wider variation across frequencies than did the perpendicular (lateral) texture.

A significant two-way interaction of texture $\times$ direction of disturbance was found $[F(4,32)=7.44, p<$

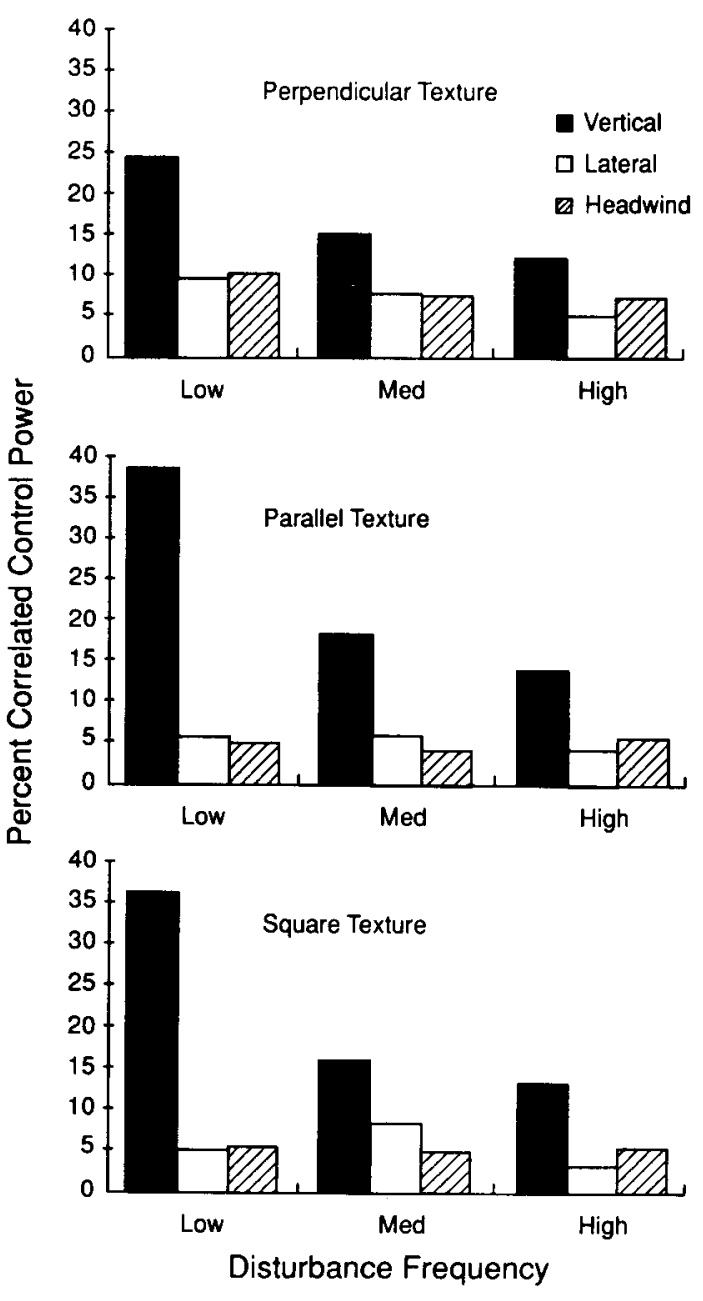

Figure 5. A significant three-way interaction between texture, frequency, and direction of disturbance was found for the response variable correlated control power in Experiment 1. 


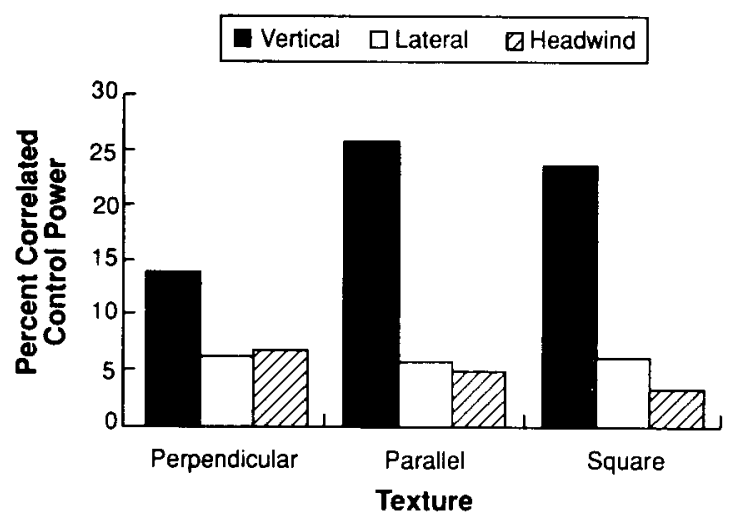

Figure 6. A significant two-way interaction between texture and direction of disturbance was found for the response variable correlated control power in Experiment 1.

.001 ], as shown in Figure 6. The levels of power associated with lateral and head-wind disturbances were similar across the texture types. However, the parallel and square textures had more power associated with vertical disturbances than did the perpendicular (lateral) texture.

A significant two-way interaction was also found for direction and frequency of disturbance $[F(4,32)=15.73$, $p<.001]$. No difference across frequency was found for the lateral and head-wind disturbances. However, for the vertical disturbance more power was associated with the lowest frequency than with the higher frequencies.

The correlated control power also resulted in significant main effects for frequency and direction. The effect of frequency $[F(2,16)=22.58, p<.001]$ showed the lowest frequency having more power than the medium or high frequencies, with no significant difference for the latter two. The effect of direction $[F(2,16)=18.28, p<$ $.001]$ showed significantly higher power associated with the vertical disturbance than with either lateral or headwind disturbances. No difference in power was found between lateral and head-wind disturbances.

\section{Discussion}

The results show clear, qualitative performance differences associated with the type of texture. Perpendicular (depression angle only) texture resulted in distinctly poorer performance than did either parallel (splay angle only) or square texture. It resulted in higher errors (mean and standard deviation), more effort (RMS control), and less precision of control (low correlated power and high residual power). No significant differences were found for parallel versus square texture. However, performance was nominally superior for parallel texture on every performance measure. These results are consistent with the results of Warren (1988), Wolpert (1988), and Wolpert et al. (1983). They suggest that optical splay angle is the more effective source of information for altitude control.

The results are clearly inconsistent with the results found by Johnson et al. (1988). Johnson et al. found su- perior performance with perpendicular texture, whereas the current results showed superior performance with parallel texture. A critical difference may have been the global optical flow rates. Johnson et al. used a hover task, whereas a continuous motion at 1 eyeheight/sec was simulated in our study. This point will receive extensive discussion at the conclusion of the paper.

Two other differences between our results and those of Johnson et al. (1988) should be considered. Johnson et al. found control power associated with vertical disturbances in the range of $50 \%-60 \%$. The current study found control power associated with vertical disturbances in the range of $3 \%-11 \%$. This difference may reflect the wider spread of frequencies at which the disturbance was input in the Johnson et al. study, 12 frequencies instead of 3 frequencies in the present study. It also may reflect the different subject populations. The present study used undergraduates. The Johnson et al. study used the best 5 of 9 subjects who volunteered for the study. Of these 5,2 were rated pilots. Johnson et al. also trained their subjects with feedback. No feedback was used in the current study. In this study, we were interested in people's naive response to the instruction to maintain altitude. Feedback with regard to error not present in the visual display itself (a score given at the end of the trial) may change the way information is extracted from the display. Finally, the differences may be due to the quality of controller used in the studies. Johnson et al. used an analog joystick, whereas we used a mouse controller. The mouse has a significant effective time delay in comparison to an analog stick. This effective time delay would explain the severe drop-off in control power across frequency seen in Figure 5.

A second difference in results was that Johnson et al. (1988) found evidence for significant amounts of crossfeed (control power) resulting from the lateral disturbance when parallel texture was present. No such evidence was found in this study; however, this may be due to a floor effect because of the relatively low power, even at the vertical disturbance frequencies.

\section{EXPERIMENT 2}

A second experiment was conducted in an attempt to replicate the results from Experiment 1. Experiment 2 was identical to Experiment 1 except that the eyeheight of the observer was doubled. Thus, global optical flow rate was reduced from 1.0 to 0.5 eyeheight/sec, and global optical density was increased to 1 ground unit/eyeheight. Thus, the distance between texture units changed from 2 eyeheights to 1 eyeheight.

\section{Method}

Subjects. Nine new subjects participated in this experiment. The subjects were University of Illinois undergraduate students participating for course credit. All had normal or corrected-to-normal vision.

Design. The design was identical to that in Experiment 1. Texture and dominant altitude frequency were manipulated within sub- 
jects across trials. These independent variables were associated with macroscopic performance variables of mean altitude error, standard deviation of altitude, residual power, and RMS stick input. The microscopic performance measure of correlated control power was affected by the independent variables: texture, direction, and frequency combined in a $3 \times 3 \times 3$ repeated measures factorial design.

Apparatus. The apparatus and stimuli were identical to those in Experiment 1 except that eyeheight was doubled. This resulted in a global optical flow rate of 0.5 eyeheight/sec and a global optical density of 1 ground unit/eyeheight. The splay angle for the nearest parallel texture unit was $26.6^{\circ}$

Procedure. The procedure was identical to that in Experiment 1.

\section{Results}

Macroscopic. As in Experiment 1 , a $3 \times 3$ repeated measures ANOVA (texture $\times$ dominant altitude frequency) was performed for each of the macroscopic performance variables.

A main effect of texture was found for mean altitude error $[F(2,16)=4.14, p<.05]$. There was greater error for the perpendicular (depression angle only) texture $(+0.69$ eyeheight) than for either the parallel (splay only) texture $(+0.32$ eyeheight) or square texture $(+0.44$ eyeheight). A similar pattern of effects was found for the adjusted mean altitude error measure.

A main effect of texture was also found for the standard deviation of altitude $[F(2,16)=10.66, p<.005]$. Greater standard deviations were found with the perpendicular texture ( 0.44 eyeheight) than with either the parallel ( 0.22 eyeheight) or the square ( 0.23 eyeheight) textures. There was only a marginal effect of texture for the adjusted standard deviation measure $[F(2,16)=2.37$, $p<.2]$. The adjusted standard deviation was 0.09 eyeheight for the perpendicular texture, 0.08 eyeheight for the parallel texture, and 0.08 eyeheight for the square texture. There was a significant main effect of dominant frequency $[F(2,16)=73.91, p<.001]$. Greater adjusted standard deviations were found for the low dominant altitude frequency ( 0.11 eyeheight) than for the medium ( 0.08 eyeheight) or high ( 0.07 eyeheight) frequencies.

No significant effects were found for the dependent measures residual stick power or RMS control.

Microscopic. A $3 \times 3 \times 3$ repeated measures ANOVA (texture $X$ direction $x$ frequency) was performed for the dependent measure correlated control power. As in Experiment 1 , a significant three-way interaction $[F(8,64)=$ $2.56, p<.05]$ was obtained. This interaction, shown in Figure 7, was similar in pattern to the effects obtained in Experiment 1.

A significant two-way interaction of texture $x$ direction of disturbance was found $[F(4,32)=4.09, p<.01]$, as shown in Figure 8. There is a crossover interaction. The perpendicular texture had less power at the vertical frequencies, but more power at the lateral and head-wind frequencies. The separation across textures for the vertical disturbance was less than that found in Experiment 1.

A significant two-way interaction was found for frequency by direction $[F(4,32)=4.58, p<.01]$, and a significant main effect was found for frequency $[F(2,16)$

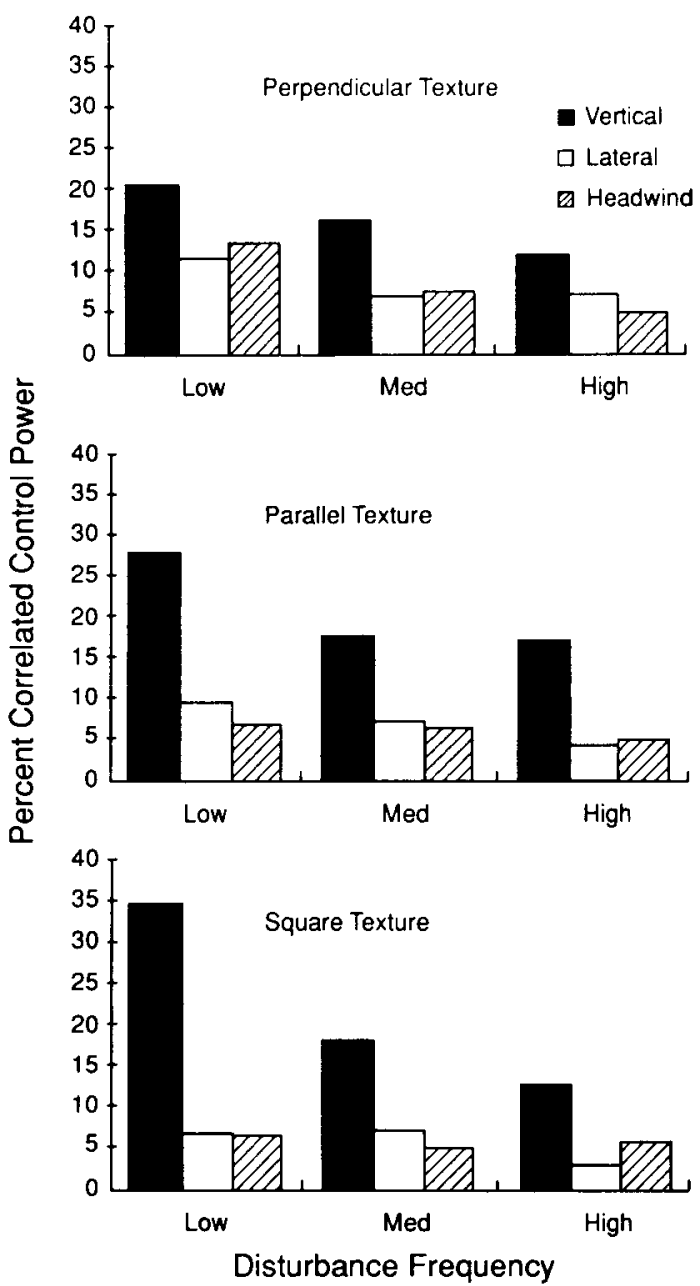

Figure 7. A significant three-way interaction between texture, frequency, and direction of disturbance was found for the response variable correlated control power in Experiment 2.

$=17.1, p<.001]$. Both effects can be seen in Figure 9. Greater power was associated with low frequency than with medium or high frequency. The interaction results because the difference across frequencies was greater for the vertical disturbance.

\section{Discussion}

The pattern of effects for Experiment 2 was in general agreement with the results from Experiment 1. However, the difference between performance with the perpendicular (depression angle only) and parallel (splay angle only) textures was somewhat attenuated for the 0.5 -eyeheight/sec global optical flow rate used in Experiment 2.

One interesting effect in Experiment 2 was the dissociation of the effects for standard deviation altitude and adjusted standard deviation altitude. The adjusted standard deviation was the median of 16 standard deviation scores, each computed over different short segments of the trial. The adjusted measure minimizes the contribution of slow 


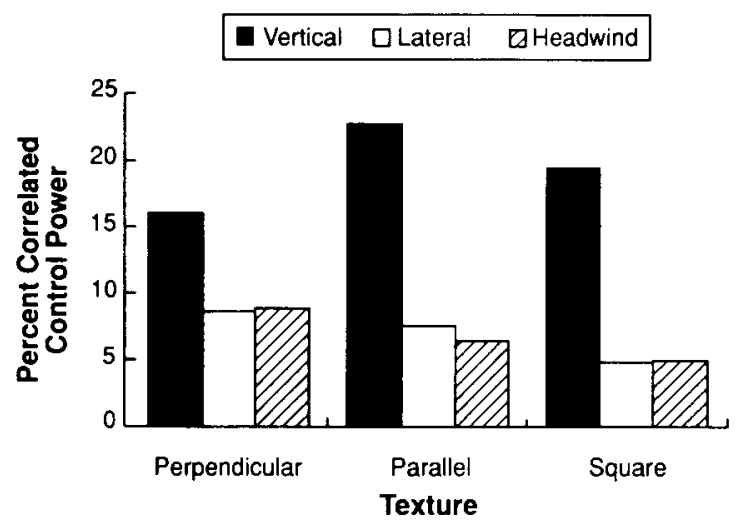

Figure 8. A significant two-way interaction between texture and direction of disturbance was found for the response variable correlated control power in Experiment 2.

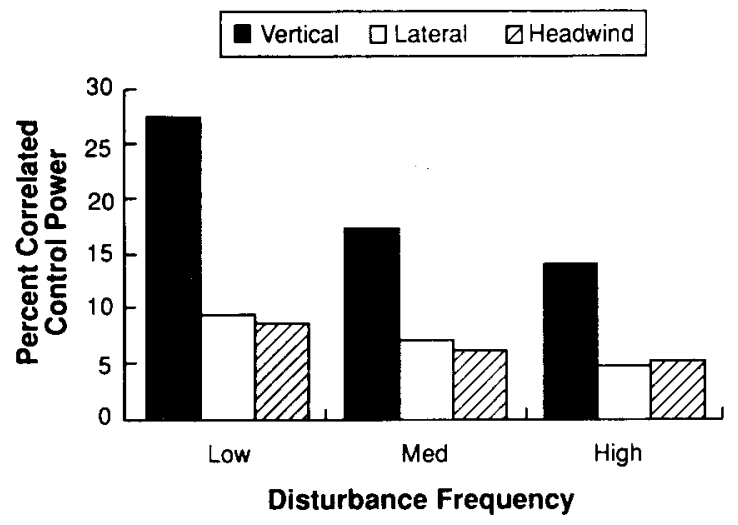

Figure 9. A significant two-way interaction between frequency and direction of disturbance was found for the response variable correlated control power in Experiment 2.

drifts to the standard deviation score (e.g., as might result from forgetting what the original altitude was and tracking a higher altitude). As Warren (1988) noted, this task is in effect "a memorial tracking task since the subjects had no way of knowing if they were on target except by 'comparing' the current state of a scene with their memory for what the scene should look like at the assigned altitude" (p. A120). The pattern of dissociation of the two standard deviation measures suggests that the parallel (splay) texture provides a more effective memorial representation. This is due to the larger standard deviation found for the perpendicular screen, showing that this texture was more susceptible to memorial drift.

\section{GENERAL DISCUSSION AND CONCLUSION}

Previous research on the optical basis for altitude control has been framed in the context of the question: Which is a more effective source of information for altitude control, optical splay angle (operationalized as texture parallel to the direction of motion) or global optical density (operationalized as texture perpendicular to the direction of motion)? The results with regard to this question have been mixed. This suggests that perhaps the question has been framed inappropriately. Perhaps the key variable is not the kind of texture (splay vs. density or perpendicular vs. parallel), but rather the amount of optical activity associated with changes in altitude (signal) relative to the amount of activity associated with changes other than altitude (noise). This suggests that neither kind of texture is privileged with regard to altitude control. The relative amount of optical activity specific to change of altitude depends both on the type of texture and on the nature of the event (e.g., forward flight or hover).

Wolpert et al. (1983) found that parallel (splay) texture resulted in better detection of loss of altitude than did square or perpendicular texture. In that study, the only optical activity in parallel texture was that associated with changes in altitude (signal). The other two textures had optical activity as a result of both changes in altitude (signal) and forward motion (noise).

Wolpert and Owen (1985) found a decrease in ability to detect loss in altitude over square texture as a function of global optical flow rate. As optical activity resulting from forward motion (noise) increased, sensitivity to altitude changes (signal) decreased.

Warren (1988) found decreased control error moving from dot, to dot and parallel texture, to parallel texture only. In the dot display, there is change in optical density associated with altitude changes (signal) and global optical flow (at high rates) associated with forward motion. Either small signal or large noise results in low sensitivity. For the dot and parallel texture display, splay information associated with changes in altitude increases signal strength, thus leading to improved performance. Finally, in the parallel texture only display, all noise associated with forward motion is removed, so that all optical activity is associated with change in altitude.

Wolpert (1988) added a roll disturbance. This produced comparable amounts of optical activity for parallel and vertical textures. Thus, while noise is added to all three types of texture, the relative ordering in terms of signalto-noise ratio is identical to that of Wolpert et al. (1983). Thus, similar results were found.

Johnson et al. (1989) used very low levels of global optical flow rate. This reduces the amount of optical activity (noise) resulting from forward motion in the perpendicular and square textures. They also introduced a lateral disturbance, which adds optical activity (noise) to the parallel and square textures. Thus, the result may be that the signal-to-noise ratio is now greater for perpendicular texture than for parallel texture. Thus, subjects are more sensitive to changes in altitude with the perpendicular texture.

Johnson et al. (1988) examined altitude control for a hover task. There was no continuous forward motion. However, a fore-aft and a lateral disturbance were added in addition to the vertical disturbance. The fore-aft disturbance added optical activity for displays with perpendicular texture, but was transparent in parallel-textureonly displays. Conversely, lateral disturbances result in 
increased optical activity (noise) for parallel texture, but are transparent in perpendicular-texture-only displays. The superior performance for perpendicular texture suggests that greater noise results from the side-to-side (lateral) disturbance. This would be consistent with an optical analysis. The affects of fore-aft disturbances on perpendicular texture are minimal near the horizon (small depression angles) and increase to a maximum under the observer (Equation 5). Most of the perpendicular elements in the field of view are near the horizon (low optical activity). However, with splay angle, changes associated with lateral disturbances are maximal at $0^{\circ}$ splay angle (Equation 2). Thus, high optical activity will be associated with the parallel elements in the field of view.

Finally, in the current study, a task very similar to that used by Johnson et al. (1988) was employed. However, forward motion was added. This resulted in optical activity that increased due to forward motion (noise) for perpendicular texture but was transparent for parallel texture. The result was superior performance with the parallel texture. However, the advantage of parallel texture was diminished when the optical flow rate (noise) was reduced from 1 eyeheight/sec in Experiment 1 to 0.5 eyeheight/sec in Experiment 2.

Thus, we can now return to the questions raised in the introduction. Could an interaction between global optical flow and the other sources of information for altitude (optical splay angle and optical depression angle) account for the disparate findings described above? The available evidence seems to indicate that the answer to this question is yes. A comprehensive study that will evaluate the relative contributions of optical splay and optical depression angle over a complete range of global optical flow rates (from 0 to greater than 1 eyeheight/sec) is currently planned.

Why does the combination of multiple sources of information (e.g., square texture) result in performance degradation? It appears that in combining the two textures, optical noise (i.e., optical activity not associated with changes in altitude) as well as additional information about altitude is added. The degradation could reflect the increase in noise.

The flip-flop in the functional value of visual information that occurs as a function of the dynamics of the various events that have provided the context for studying altitude regulation is not unprecedented. A similar flip-flop is reported for judgment of slant. For judgment of slant in static displays, perspective dominates form ratio. However, for judgment of slant in dynamic displays, form ratio is clearly dominant (Braunstein, 1976). Thus, the functional value of visual information must be considered in the context of an event. Important aspects of events relevant for altitude control appear to be whether the observer is translating or hovering and whether disturbances are present on axes other than the vertical axes.

An important aspect of dynamics that has not been varied is the ability to control axes other than the vertical. In all the studies reviewed here, the observer had control only over altitude. All other axes (i.e., forward, lateral, and roll motion) were controlled by the experimenters. It will be important for future studies to evaluate whether the effects due to "optical noise" on these axes change when the observer has active control over disturbances on these axes.

In summary, there are at least two factors to consider in comparing the alternative textures (splay vs. optical depression angle) in terms of their effectiveness for perception. The first factor is the specificity of the optical texture to the experimental task. Our analysis suggests that optical depression angle is specific to altitude control only under the very special case of hovering, but that splay angle may be more specific under the more general case of straight and level flight. The other factor is how well the visual system is tuned to the various textures. The fact that there is an apparant interaction as a function of task constraints suggests that there is some degree of flexibility within the perceptual system. However, it may be important to know which kind of texture is most natural or is preferred. To address this issue, one strategy might be to find a task context that is neutral in terms of specificity. That is, the signal-to-noise ratio must be equated for splay and for optical depression angle. On the other hand, it might be argued that the perceptual system is not differentially tuned as a function of texture type, but in fact may be designed simply to resonate to specificity. If this is the case, then understanding the specificity of information in the context of tasks may be fundamental to a theory of perception.

\section{REFERENCES}

ATtNeAve, F., OLSON, R. K. (1966). Inferences about visual mechanisms from monocular depth effects. Psychonomic Science, 4, 133-134.

BigGs, N. L. (1966). Directional guidance of motor vehicles: A preliminary survey and analysis. Ergonomics, 9, 193-202.

Braunstein, M. L. (1976). Depth perception through motion. New York: Academic Press.

Cutting, J. E., Millard, R. T. (1984). Three gradients and the perception of flat and curved surfaces. Joumal of Experimental Psychology: General, 113, 198-216.

Gibson, J. J., Olum, P., Rosenblatt, F. (1955). Parallax and perspective during aircraft landings. American Journal of Psychology, $68,372-385$.

GILLAM, B. J. (1968). Penception of slant when perspective and stereopsis conflict: Experiments with aniseikonic lenses. Joumal of Experimental Psychology, 78, 299-305.

GillaM, B. J. (1970). Judgments of slant on the basis of foreshortening. Scandinavian Journal of Psychology, 11, 31-34.

Johnson, W. W., Bennett, C. T., O'Donnell, K., Phatak, A. V. (1988). Optical variables useful in the active control of altitude. Paper presented at the 23rd Annual Conference on Manual Control, Cambridge, MA.

Johnson, W. W., Tsang, P. S., Bennett, C. T., \& Phatak, A. V. (1989). The visually guided control of simulated altitude. Aviation, Space, \& Environmental Medicine, 60, 152-156.

LARISH, J. F., FLACH, J. M. (1990). Sources of optical information useful for perception of speed of rectilinear self-motion. Journal of Experimental Psychology: Human Perception \& Performance, 16, 295-302.

LEE, D. N. (1976). A theory of visual control of braking based on information about time to collision. Perception, 5, 437-459. 
LEE, D. N. (1980). The optical flow field: The foundation of vision. Philosophical Transactions of the Royal Society of London: Series B, 290, 169-178.

Rosinski, R. R., Levine, N. P. (1976). Texture gradient effectiveness in the perception of surface slant. Joumal of Experimental Child Psychology, 22, 261-271.

VICKERS, D. (1971). Perceptual economy and the impression of visual depth. Perception \& Psychophysics, 10, 23-27.

WARREN, R. (1982). Optical transformation during movement: Review of the optical concomitants of egomotion. (NTIS Tech. Rep. No. ADA122275). Columbus, OH: Ohio State University, Department of Psychology.

WARREN, R. (1988). Visual perception in high-speed low altitude flight. Aviation, Space, \& Environmental Medicine, 59(Suppl. 11), A116-A124.

WOLPERT, L. (1983). The partitioning of self-scaled and texture-scaled optical information for detection of descent. Unpublished master's thesis, Ohio State University, Columbus, $\mathrm{OH}$.

WOLPERT, L. (1987). Field of view versus retinal region in the perception of self-motion. Unpublished doctoral dissertation, Ohio State University, Columbus, $\mathrm{OH}$.

WOLPERT, L. (1988). The active control of altitude over differing texture. In Proceedings of the 32nd Annual Meeting of the Human Factors Society (pp. 15-19). Santa Monica, CA: Human Factors Society.

WOLPERT, L., \& OWEN, D. (1985). Sources of optical information and their metrics for detecting loss in altitude. In Proceedings of the 3rd
Symposium on Aviation Psychology (pp. 475-481). Columbus, OH: The Ohio State University.

WolPERT, L., OWEN, D., WARREN, R. (1983). Eye-height-scaled versus ground-texture-unit-scaled metrics for the detection of loss in altitude. In Proceedings of the 2nd Symposium on Aviation Psychol$o g y$, pp. 513-521). Columbus, OH: The Ohio State University.

\section{NOTES}

1. To understand this, view each of the textures in Figure 2 through the hole in a sheet of loose-leaf paper. Now move the texture from side to side behind the hole. Note that the side-to-side motion is functionally invisible with respect to perpendicular texture.

2. Because of publication lags, the dates do not reflect the sequence in which these experiments were performed. The 1989 study was done prior to the 1988 study.

3. Again, to convince yourself, you can view the textures in Figure 2 through the hole in a piece of loose-leaf paper and roll the texture beneath the paper.

4. The simulated altitude was $320 \mathrm{~m}$, and the initial forward velocity was $320 \mathrm{~m} / \mathrm{sec}$. However, throughout this report, the intrinsic scale that employs eyeheights as a metric will be used. This focuses attention on the optical transformations.

(Manuscript received September 15, 1989 ; revision accepted for publication January 8, 1992.) 